\title{
Reverse takotsubo cardiomyopathy: a rare complication of epidural steroid injection
}

\author{
Volume 6 Issue 6 - 2017
}

\begin{abstract}
Stress-induced cardiomyopathy, also known as Takotsubo cardiomyopathy (TCMP) is characterized by a rapid, severe and usually reversible ventricular wall dysfunction, ST segment changes on EKG and troponin leak without evidence of obstructive coronary artery disease by coronary angiography. It represents approximately $1.2 \%$ of all troponin positive acute coronary syndromes. ${ }^{1}$ Physical or emotional stressors have been identified as triggers precipitating this syndrome in the majority of the cases.

Classification of TCMP is based on the distribution of wall motion abnormalities noted on Echocardiogram. Study of International Takotsubo Registry (1750 patients) identified 4 types of TCMP, apical (81.7\%), midventricular (14.6\%), basal/reverse $(2.2 \%)$ and focal type $(1.5 \%) .^{2}$ These patterns of myocardial dysfunction are reminiscent of myocardial stunning associated with an increased catecholamine release. It has been hypothesized that the distribution of adrenergic receptors in myocardial tissue explains the difference in anatomical distribution of injury. ${ }^{3}$ Here we present a case of a middle-aged female patient who developed acute severe heart failure caused by reverse TCMP following a cervical steroid injection.

With this case report, we intend to highlight the possible association between epidural steroid injection and development of TCMP. We also emphasize the high clinical suspicion required to diagnose TCM in patients who present with acute coronary syndrome, heart failure, specific pattern of wall motion abnormalities and angiographically normal coronaries. We further illustrate the utility of cardiac MRI as a non-invasive study with increased sensitivity over other imaging modalities in diagnosing TCM.
\end{abstract}

\author{
Nino Isakadze,' Beka Bakhtadze,' Nikolaos \\ Spilias,' Susmita Parashar ${ }^{2}$ \\ 'Division of Medicine, Emory University School of Medicine, \\ USA \\ ${ }^{2}$ Division of Cardiology, Emory University School of Medicine,
} USA

Correspondence: Nino Isakadze, Division of Medicine, Emory University School of Medicine, USA,

Email: ninoisakadzee@gmail.com

Received: May 26, 2017| Published: June 12, 2017

\section{Case presentation}

A highly functional middle-aged postmenopausal female with remote history of depression, cervical canal stenosis and no other significant medical history presented to the emergency room after awakening around midnight with severe headache, diaphoresis, palpitations, shortness of breath, nausea, vomiting and diarrhea. The patient was severely hypertensive upon presentation. At presentation she had severely elevated blood pressure (BP 220/150mm Hg), was diaphoretic and complained of severe sub sternal non-radiating chest pain. Physical examination revealed regular rhythm, with a heart rate of 122 beats per minute, mild respiratory distress and she was afebrile. Her jugular venous pressure was not elevated and no carotid bruits were present. Cardiac auscultation revealed normal first and second heart sounds with a $2 / 6$ holosystolic murmur at the apex radiating to axilla. Her peripheral pulses were equal in all extremities. Lung fields were clear. She denied any acute stressors. Patient reported cervical spinal pain for which she had received a cervical steroid injection several hours before presentation.

\section{Investigations}

Due to her complains of chest pain, a stat electrocardiogram (EKG) was obtained. Initial EKG showed fascicular ventricular tachycardia with HR of 148 beats per minute, which subsided with 5 $\mathrm{mg}$ of IV Metoprolol. Subsequent EKG showed new 2mm ST segment depressions in II, III, AVF and precordial leads, and incomplete right bundle branch block. Laboratory studies were notable for troponin-I of $1.19 \mathrm{ng} / \mathrm{mL}$. A stat cardiology consultation was obtained. An urgent bedside echocardiogram demonstrated reduced ejection fraction of $25-30 \%$ and regional wall motion abnormalities localized in the basal segments. The apex of the heart was contracting normally while all the basal segments were severely hypokinetic. An emergent cardiac catheterization showed no significant epicardial coronary artery stenosis. Left ventricular end diastolic pressure was $33 \mathrm{~mm} \mathrm{Hg}$. Left ventriculogram showed systolic hypokinesis of the entire base with hypercontractile apex and LVEF of 30\%. A diagnosis of TCMP was made due to the clinical presentation with EKG findings and wall motion abnormality in the absence of coronary stenosis.

Subsequent work up demonstrated a sedimentation rate of $10 \mathrm{~mm} /$ $\mathrm{hr}$ and an elevated C-reactive protein $(27.6 \mathrm{mg} / \mathrm{L})$. Peak troponin was 17.6ng/mL. Cocksackie B1 titer was 1:160 (normal <1:10). A cardiac MRI was performed 3 weeks after discharge to distinguish between myocarditis and TCMP, which showed mild residual hypokinesis of the basal segments of the left ventricular myocardium, consistent with resolving reverse Takotsubo cardiomyopathy. There were no abnormalities for gadolinium enhancement to suggest ischemic etiology or myocarditis.

\section{Treatment}

Given this finding by echocardiography along with normal coronary angiography, patient was treated supportively with diuretics and angiotensin-converting enzyme inhibitor. Patient did not require diuretics upon discharge. Beta blockers could not be started due to bradycardia. 


\section{Outcome and follow-up}

Patient had excellent recovery; she started Yoga and gradually increased physical activity. Repeat echocardiogram performed after 6month showed resolved wall motion abnormalities and ejection fraction of $60 \%$. This improvement in imaging correlated well with her clinical cardiac improvement.

\section{Discussion}

Takotsubo cardiomyopathy (TCMP) is a condition characterized by profound, yet reversible acute heart failure with possible lifethreatening complications. Reverse takotubo cardiomyopathy is a subtype of TCMP. Acute emotional or physical stress has historically described as triggering factor for this syndrome. ${ }^{1}$ TCMP mostly affects women, who represent majority (89\%) of all patients diagnosed with TCMP. ${ }^{4}$ It has been reported that older females are more vulnerable to stress induced cardiomyopathy, this is hypotehesized to be secondary to reduction of estrogen levels following menopause that can lead to takotsubo cardiomyopathy both by indirect action on the nervous system and by direct action on the heart. ${ }^{4}$ Patients typically present with acute substernal chest pain accompanied by dyspnea, syncope, or shock. Electrocardiographic changes usually include initial ST segment elevation followed by $\mathrm{T}$ wave inversion with QT prolongation throughout the anterior and precordial leads. ${ }^{5,6}$ Rarely, the patients can develop ventricular arrhythmias, such as VT or ventricular fibrillation. Cardiac enzymes are typically elevated, it represents approximately $1.2 \%$ of all troponin positive coronary syndromes. ${ }^{1}$ Cardiac MRI allows differentiating TCMP from other rare entities with negative coronary angiogrpaphy such as myocarditis and coronary emboli with spontaneous lysis. Cardiac MRI reveals hypokinetic or akinetic basal segments with apical sparing and subendocardial edema without evidence of fibrosis or necrosis, corresponding to the affected segments (different from myocarditis pattern, which has more subepicardial preference for the myocardial edema). ${ }^{8}$ Therefore, cardiac MRI can add valuable information in all patients with suspected TCMP for further differential. ${ }^{9}$ The prognosis is favorable. Pilgrim et al. ${ }^{10}$ demonstrated in a systemic review summarizing 28 case series that mortality rate was $1.7 \%$ with complete recovery in $95.5 \%$ and risk of recurrence was very low. ${ }^{10}$ Another review by Gianni et al reviewing 14 studies showed very similar mortality rate- $1.1 \%$ with only $3.5 \%$ of the patients experiencing a recurrence. ${ }^{4}$

Per recent review of 79 patients with TCMP, only $48 \%$ of cases had clearly identified trigger. ${ }^{11}$ In addition to emotional and physical stress subarachnoid hemorrhage, cerebellar hemorrhage, diving, iatrogenic epinephrine use, epidural neuroplasty have been reported as precipitants of TCMP among others. ${ }^{5,12-14}$ To the best of our knowledge there is only one case report of TCMP, specifically apical type TCMP following an thoracic epidural steroid injection. We describe first case of reverse TCMP following cervical epidural steroid injection.

Basal/reverse type of Takotsubo cardiomyopathy represents only $2.2 \%$ cases of TCMP. ${ }^{2}$ It is characterized by echocardiographic findings of basal inferior wall hypokinesis. Interestingly prevalence of basal/reverse type is significantly higher in younger female patients as compared to postmenopausal females for apical TCMP. Hypothesis exists that above observation is because younger patients have higher concentration of adrenoreceptros at the base of the heart, compared to number of apical receptors in older patient population. ${ }^{15}$ Recognizing TCMP (revere or any other type) as a potential complication for epidural steroid injection is important as currently these procedures are one of the most commonly performed medical procedures in the United States, especially in pain medicine clinics. ${ }^{16}$ Rapid recognition of this syndrome may help to identify patients with warning symptoms early and lead to prompt initiation of medical care.

Interestingly our patient and patient described in the recent case report with TCMP following thoracic steroid injection had very similar presentation. ${ }^{17}$ Both were female, postmenopausal, with history of chronic stress, both underwent steroid injection uneventfully with development of severe headache and progressively worsening chest pain following several hours. Data is still anecdotal, however our case report along with recently published case of TCMP following epidural steroid injection supports hypothesis that females with history of depression may represent vulnerable population with increased risk of TCMP. We believe that our patient's chronic stress predisposed her to TCMP and epidural injection acted as trigger. ${ }^{3}$

\section{Learning points}

i. Epidural steroid injection may act as precipitating factor for takotsubo cardiomyopathy.

ii. Patients with Takotsubo cardiomyopathy despite dramatic initial presentation, have overall good prognosis.

iii. Cardiac MRI is useful to differentiate Takotsubo cardiomyopathy from Myocarditis.

\section{Acknowledgements}

None.

\section{Conflict of interest}

The author declares no conflict of interest.

\section{References}

1. Templin C, Ghadri JR, Diekmann J, et al. Clinical features and outcomes of takotsubo (Stress) cardiomyopathy. N Engl J Med. 2015;373(10):929938

2. Kurowski V, Kaiser A, von Hof K, et al. Apical and midventricular transient left ventricular dysfunction syndrome (tako-tsubo cardiomyopathy):frequency, mechanisms, and prognosis. Chest. 2007;132(3):809816.

3. S YH, De Palma R. Contemporary review on the pathogenesis of takotsubo syndrome: The heart shedding tears: Norepinephrine churn and foam at the cardiac sympathetic nerve terminals. Int $J$ Cardiol. 2017;228:528-536.

4. Gianni M, Dentali F, Grandi AM, et al. Apical ballooning syndrome or takotsubo cardiomyopathy: a systematic review. Eur Heart J. 2006;27(13):1523-1529.

5. Baber A, Nair SU, Duggal S, et al. Stress Cardiomyopathy caused by diving: case report and review of the literature. $J$ Emerg med. 2015;50(2):277-280.

6. Mitsuma W, Kodama M, Ito M, et al. Serial electrocardiographic findings in women with Takotsubo cardiomyopathy. Am $j$ cardiol. 2007;100(1):106-109.

7. Senecal EL, Rosenfield K, Caldera AE, et al. Case records of the Massachusetts General Hospital. Case 36-2011. A 93-year-old woman with shortness of breath and chest pain. N Engl J Med. 2011;365(21):20212028 . 
8. Lee CH, Son JW, Kim U. Reverse Takotsubo Cardiomyopathy following Inadvertent Intrathecal Injection during Percutaneous Epidural Neuroplasty. Heart, lung \& circ. 2015;24(9):e148-e151.

9. Eitel I, Behrendt F, Schindler K, et al. Differential diagnosis of suspected apical ballooning syndrome using contrast-enhanced magnetic resonance imaging. Eur Heart j. 2008;29(21):2651-2659.

10. Pilgrim TM, Wyss TR. Takotsubo cardiomyopathy or transient left ventricular apical ballooning syndrome: A systematic review. Int J cardiol. 2008;124(3):283-292.

11. Glaveckaite S, Serpytis P, Peciuraite D, et al. Clinical features and threeyear outcomes of Takotsubo (stress) cardiomyopathy:Observational data from one center. Hellenic J Cardiol. 2016;57(6):428-434.

12. Pierard S, Vinetti M, Hantson P. Inverted (Reverse) Takotsubo cardiomyopathy following cerebellar hemorrhage. Case reports in cardiology. 2014;2014:781926.

13. Litvinov IV, Kotowycz MA, Wassmann S. Iatrogenic epinephrine-induced reverse Takotsubo cardiomyopathy: direct evidence supporting the role of catecholamines in the pathophysiology of the "broken heart syndrome". Clinical research in cardiology: official journal of the German Cardiac Society. 2009;98(7):457-462.
14. Waller CJ, Vandenberg B, Hasan D, et al. Stress cardiomyopathy with an "inverse" takotsubo pattern in a patient with acute aneurysmal subarachnoid hemorrhage. Echocardiography. 2013;30(8):E224-E226.

15. Ramaraj R, Movahed MR. Reverse or inverted takotsubo cardiomyopathy (reverse left ventricular apical ballooning syndrome) presents at a younger age compared with the mid or apical variant and is always associated with triggering stress. Congest Heart Fail. 2010;16(6):284-286.

16. Cohen SP, Bicket MC, Jamison D, et al. Epidural steroids: a comprehensive, evidence-based review. Reg Anesth Pain Med. 2013;38(3):175200 .

17. Schussler JM. Takotsubo cardiomyopathy following epidural steroid injection: yet another way to break the heart. Proc (Bayl Univ Med Cent). 2014;27(2):122. 